\title{
Retroperitoneal Liposarcoma Mimicking an Ovarian Tumor: A Diagnostic Dilemma
}

\author{
K. Harou, Y. Ouldbenazzouz*, Y. Aitbenkadour, H. Bassir, H. Asmouki, A. Soumani \\ Gynecology and Obstetrics Service chu Mohammed vi Marrakech \\ Email: *younesbenazzouz88@gmail.com
}

How to cite this paper: Harou, K., Ouldbenazzouz, Y., Aitbenkadour, Y., Bassir, H., Asmouki, H. and Soumani, A. (2018) Retroperitoneal Liposarcoma Mimicking an Ovarian Tumor: A Diagnostic Dilemma. Open Journal of Obstetrics and Gynecology, 8, 288-292.

https://doi.org/10.4236/ojog.2018.83030

Received: November 5, 2017

Accepted: March 27, 2018

Published: March 30, 2018

Copyright (๑) 2018 by authors and Scientific Research Publishing Inc. This work is licensed under the Creative Commons Attribution-NonCommercial International License (CC BY-NC 4.0).

http://creativecommons.org/licenses/by-nc/4.0/

\begin{abstract}
Introduction: Retroperitoneal liposarcoma is a malignant, primitive and rare mesenchymal tumor. We report an observation of a retroperitoneal liposarcoma mimicking an ovarian tumor. Case: A 36-year-old female presented a pelvic pain type gravity whose examination found an abdominopelvic mass, an abdominopelvic computed tomography showed a large abdominal-pelvic mass with a fatty component leading to the diagnosis of ovarian teratoma. At exploratory laparotomy, we found a large abdomino-pelvic mass of $30 \times 35$ $\mathrm{cm}$, and a large resection was realised. The histopathologic examination found a liposarcoma well differentiated (grade 1). Conclusion: The different pathological aspects, the therapeutic options and the prognostic factors of the abdominal liposarcomas will be discussed in this article.
\end{abstract}

\section{Keywords}

Liposarcoma, Retroperitoneum

\section{Introduction}

Liposarcomas are malignant neoplasm deriving from the fatty tissue. There are rare tumors accounting for $0.8 \%$ to $1 \%$ of all malignant neoplasm and only $10 \%-15 \%$ are retroperitoneal [1]. They pose a problem of diagnostic and therapeutic because the symptoms are usually late and nonspecific, so they can grow slowly in the retroperitoneal space and they extend in retroperitoneal into the female pelvis that represent a potential pitfall for gynecologists who can misdiagnose them as adnexal masses. We report a case of retroperitoneal liposarcoma, mimicking an ovarian tumor due to radiological similarities.

\section{Case}

Patient aged 36, 2 gestures 2 parities 2 living children, having a regular cycle 
without notion of contraception and without any personnel or family medical history, presented since 2 months pelvic pain type gravity accompanied with abdominal distension. During examination we found a huge abdominopelvic masse arriving to umbilicus with soft consistency, mobile, sensitive to palpation and the rest of examination was normal. Ultrasonography showed a homogeneous, hyperechogenic abdomino-pelvic mass without vegetations extended to the right kidney, without peritoneal effusion and the color Doppler examination showed poor intratumor vascularization, the size of the uterus was normal and the ovaries weren't visible. Computed tomography (CT) revealed a large abdomino-pelvic mass measured $30 \mathrm{~cm} \times 40 \mathrm{~cm}$ with a fat component, leading to the diagnosis of ovarian teratoma (Figure 1). The CA125 was negative.

At exploratory laparotomy, we found a large abdomino-pelvic mass of $30 \times 35$ $\mathrm{cm}$, adherent to the aorta, inferior vena cave and blood vessels. The tumor was composed of multiple fat lobules traversed by fine septas with the involvement of the right ureter. A wide resection was realized whose the macroscopic appearance of the tumor is composed of several lobes of fatty nature measured $40 \times 45 \mathrm{~cm}$ and weighing $12 \mathrm{~kg}$ (Figure 2). In the present case, the histopathologic findings were a tumor proliferation of lipomatous nature associated with loose myxoidfoci; the border of the fragments is lesional concluding a voluminous liposarcoma well differentiated (grade 1) (Figure 3). Her postoperative course was uneventful. The patient was discharged on the $8^{\text {th }}$ postoperative day and referred for radiotherapy after multidisciplinary consultation meeting staff.

\section{Discussion}

Sarcomas are a rare mesenchymal tumors accounting for less than $1 \%$ of all malignant tumors, $10 \%$ to $15 \%$ are located in the retroperitoneum and are dominated by liposarcoma, which accounts for about $40 \%-50 \%$ of retroperitoneal sarcoma [1] [2]. Retroperitoneal liposarcoma affects both sexes equally. The average age at diagnosis is around the fifth decade, but the disease may interest all ages [2]. The increase in the size of the tumor and the complacency of the retroperitoneal space explained the asymptomatic character and the large dimensions of these tumors. In our case, the tumor was $30 \mathrm{~cm}$. The symptoms revealing the

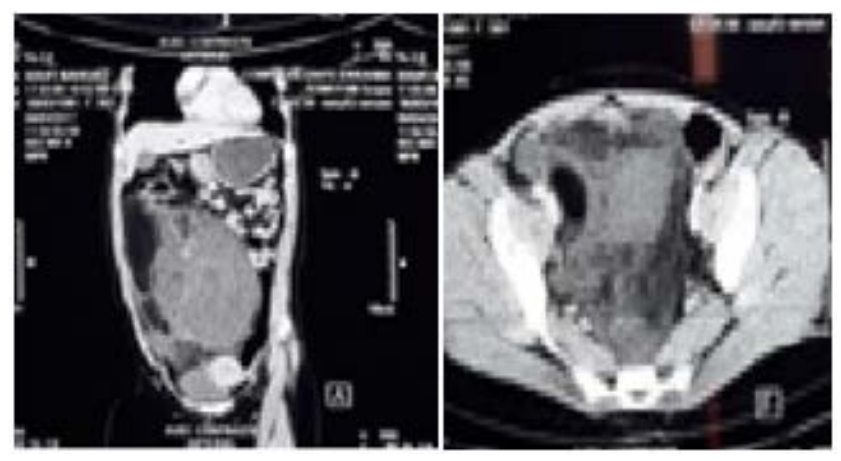

Figure 1. Computed tomography (CT) revealed a large abdomino-pelvic mass. 


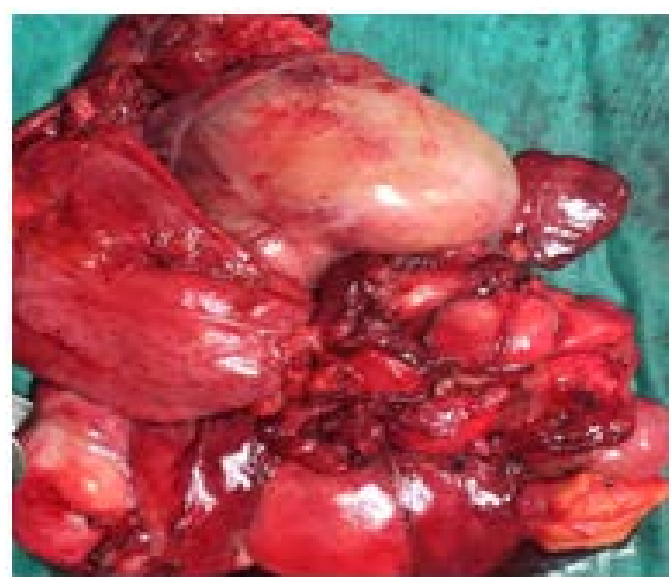

Figure 2. Macroscopic examination: polylobed mass of fatty nature.

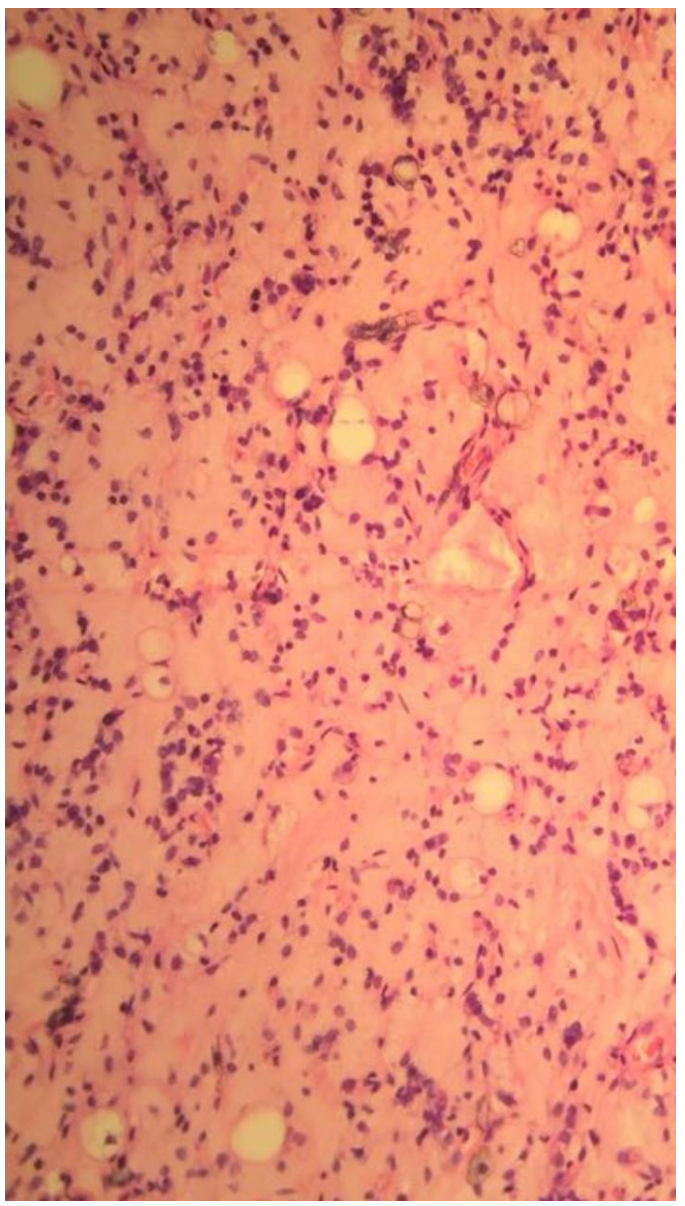

Figure 3. Malignant tumor poorly differentiated with some lipoblasts on a myxoid background.

diseases are not specific, retroperitoneal liposarcoma can be diagnosed when they reaching a considerable size or when there is an invasion or compression of the surrounding organs. Furthmore it can be revealed by complication like haemorrhage, intestinal obstruction, perforation or volvulus [2] [3]. However, lipo- 
sarcomasthat extends retroperitoneally into the female pelvis represent a potential pitfall for gynecologists, who can misdiagnose them as adnexal masses [4]. The abdominopelvic ultrasound may confirm the diagnosis in $30 \%$ to $50 \%$ of the cases. Computed tomography allows for differentiation between well differentiated liposarcomas and other tumour types on the basis of higher fat content, the presence of thick septas separating fat lobules and the absence of calcifications. Magnetic resonance imaging specifies the characteristics of the masses and may also be useful in the detection of recurrences and metastases [5]. Only the anatomopathological examination of the piece of resection tumorale allows confirming the diagnosis and making more accurate classification of the histological type of the tumour. Several histological varieties of increasing malignancy have been described. Well differentiated liposarcoma, myxoid liposarcoma, pleomorphic liposarcoma, round cell liposarcoma and dedifferentiated liposarcoma. Well differentiated and myxoid types are characterized by a low degree of malignancy though they have a tendency to recur locally (30\%). Liposarcomas with a high degree of malignancy are of the pleomorphic and poorly differentiated types. In these cases, the clinical course is much worse and metastases to the lungs are observed in $80 \%-90 \%$ of cases [6] [7]. The large excision of the tumor, with the viscera it invades, in monoblocis the reference treatment. Adequate resection margins are necessary for recovery. Complete resection is possible in $40 \%-60 \%$ of cases of retroperitoneal tumours. Recurrences are observed in $15 \%$ $36 \%$ of cases and have been described even after periods of over 10 years and up to 30 years. Liposarcomas are among the most radiosensitive of the sarcomas and radiotherapy (60 - $70 \mathrm{~Gy}$ ) may be applied before, during or after surgery [8]. Thus, overall survival is estimated to be about 50\% at five years and about $39 \%$ at ten years [9].

\section{Conclusion}

Our case shows some peculiar characteristics of giant retroperitoneal liposarcoma in a 36-years-old female measuring $30 \times 35 \mathrm{~cm}$, initially diagnosed as an ovarian tumor, based on clinical and radiologic findings. However the surgical resection is the only therapeutic way that can confirm the diagnosis and provide a concrete perspective of care. The place of radiotherapy and neoadjuvant chemotherapy is being evaluated. Gynecologists should be familiar with pelvic retroperitoneal liposarcomas and their treatment, and should pay a special attention to fertility preservation when treating young patients.

\section{References}

[1] Ameur, A., et al. (2003) Les sarcomes rétropéritoneaux: Aspects thérapeutiques et pronostiques, à propos de 6 cas. Annales d'urologie, 37, 258-263. https://doi.org/10.1016/S0003-4401(03)00095-0

[2] Antinori, A., Antonacci, V. and Magistrelli, P. (2002) Giant Retroperitoneal Liposarcoma. The American Journal of Surgery, 184, 56-57. https://doi.org/10.1016/S0002-9610(02)00880-2 
[3] Ben Moualli, S., Mnif, A., Ben Amna, M., Ben Hassine, L., Chebil, M., Zermani, A., et al. (2002) Le liposarcome rétropéritonéal géant: A propos d'un cas. Annales d Urologie, 36, 272-275. https://doi.org/10.1016/S0003-4401(02)00131-6

[4] Susini, T. (2000) Giant Pelvic Retroperitoneal Liposarcoma. Obstetrics \& Gynecology, 95, 1002-1004.

[5] Dodd, G.D. (1990) Lipomatous Tumors of the Pelvis in Women: Spectrum of Imaging Findings. AJR. American Journal of Roentgenology, 155, 317-322. https://doi.org/10.2214/ajr.155.2.2115259

[6] Maàmouri, N., et al. (2005) Liposarcome rétropéritonéal géant. À propos d'un cas. La revue de Médecine Interne, 26, 145-148. https://doi.org/10.1016/j.revmed.2004.10.029

[7] Tuoheti, Y., Okada, K., Miyakoshi, N., Nishida, J. and Itoi, E. (2002) Unusual Variant of Liposarcoma with Multiple Punctate Calcifications. Skeletal Radiology, 31, 666-670. https://doi.org/10.1007/s00256-002-0561-5

[8] Murawa, D. (2005) Cases of Giant Retroperitoneal Liposarcomas. Reports of Practical Oncology and Radiotherapy, 10, 147-151.

https://doi.org/10.1016/S1507-1367(05)71088-9

[9] Tsang, A., Nash, J.R., Fordham, M.V., Hartley, M.N. and Poston, G.J. (2003) The Management of Retroperitoneal Liposarcoma with Synchronous Intraduodenal Sarcoma. European Journal of Surgical Oncology, 29, 515-518.

https://doi.org/10.1016/S0748-7983(03)00050-7 